\section{P4.059 HIV PREVALENCE AND BEHAVIOURAL RISK FACTORS AMONG MALE SEX WORKERS (MONEY BOYS) IN AN URBAN CITY OF SOUTHWEST CHINA}

\section{doi:10.1136/sextrans-2013-051184.0957}

${ }^{1} \mathbf{B} \mathbf{Y u},{ }^{2} \mathrm{X}$ Wang, ${ }^{2} \mathrm{H} \mathrm{Xu},{ }^{3} \mathrm{~J}$ Zhang, ${ }^{3} \mathrm{X} \mathrm{Ma},{ }^{1} \mathrm{R}$ Lorway, ${ }^{1} \mathrm{~J} \mathrm{~F}$ Blanchard. ${ }^{1}$ University of Manitoba, Winnipeg, MB, Canada; ${ }^{2}$ Tongle Health Counseling Service Centre, Chengdu, China; ${ }^{3}$ Sichuan University, Chengdu, China

Background Male sex workers (MSW) in China, known as "money boys", have been identified as a bridging population for transmission of HIV/STIs between communities of men who have sex with men (MSM) and the general population.

Method Based on geographic mapping and enumeration results in Chengdu, a sample of sex work venues $(n=23)$ was selected and profiled to understand the network operation and forming of the MSW industry in Chengdu. To reduce the social desirability bias of face-to-face interviews, Polling Booth Survey (PBS) was used to document the risks behaviours of MSWs $(n=130)$. Data from voluntary counselling/testing (VCT) of MSW ( $\mathrm{N}=531)$ and general MSMs $(\mathrm{N}=1908)$ were analysed for HIV prevalence.

Results On average, MSW provided sexual services to 4-6 clients (mean $=2.4 \pm 3.5$ ) per day, with as many as 10 clients (mean $=7.1 \pm$ $5.25 \mathrm{std}$ ) in a peak day. All respondents reported $100 \%$ condom use during face-to-face interviews. PBS results, however, revealed that only $74 \%$ of MSW used condoms every time and $76 \%$ in last anal sex. Using condoms with permanent sex partners was lower $(44 \%$ last month anal sex). 14\% of MSW had anal sex $3+$ times, and $4 \%$ had anal sex $7+$ times in one day. VCT data showed a relatively lower HIV prevalence $(6 \%)$ in MSW $(\mathrm{N}=531)$ compared to the HIV prevalence $(10 \%)$ in MSM in 2012. 29\% of MSWs identified as heterosexual, $20 \%$ as bisexual, and $51 \%$ as gay.

Conclusion Male sex work in urban China has grown into a thriving industry. Due to high client volumes, unprotected anal sex, multiple concurrent sexual partners, and participation in sexual networks that span various cities throughout China, MSWs are at a higher risk for HIV/STI transmission. PBS is a more reliable method to examine risky sexual behaviours among high risk populations.

\section{P4.060 ACCURACY AND CORRELATES OF INFIDELITY SUSPICIONS AMONG MARRIED COUPLES IN THE FISHING COMMUNITIES ON LAKE VICTORIA IN KISUMU COUNTY, KENYA}

doi:10.1136/sextrans-2013-051184.0958

'Z A Kwena, ${ }^{2} \mathrm{C}$ A Shisanya, ${ }^{2}$ Mwanzo, 'E A Bukusi. 'Kenya Medical Research Institute, Kisumu, Kenya; ${ }^{2}$ Kenyatta University, Nairobi, Kenya

Background Inaccurate suspicions of infidelity among married couples can affect trust and intimacy in a relationship. We sought to establish the prevalence, accuracy and correlates of infidelity suspicions among married couples.

Methods We conducted a cross-sectional survey of 545 couples. We contacted a proportion to size simple random sample of fishermen on each beach as our index participants and asked them to enrol with their spouses. The consenting couples were separated into different private rooms for simultaneous gender-matched interviews. In addition to socio-economic and behavioural data, we collected information on extra-marital sex partners and infidelity suspicions among spouses. We used descriptive statistics and multivariate logistic regression for analysis.

Results A quarter (27.2\%) of the women and $16.9 \%$ of the men suspected their spouses of infidelity but only $6.2 \%$ of the women and $32.1 \%$ of the men reported extra-marital relationship. Overall, $43.2 \%$ of the women and $6.5 \%$ of the men accurately suspected their spouses with sensitivity of $36.6 \%$ and $17.6 \%$ respectively. Correlates of women accurately suspecting their spouses were: men being mobile (OR, 0.58; 95\% CI: 0.39-0.85), couple being in polygamous marriage (OR, 0.48; $95 \% \mathrm{CI}: 0.25$ 0.90 ), taking longer time during sexual intercourse (OR, 0.97; 95\% CI: 0.95-0.99), men's higher score on male dominance sex roles (for all our scales, higher score better) (OR, 1.08; 95\% CI: 1.03-1.13) and higher score on domestic violence scale (OR, 1.27; 95\% CI: 1.12-1.44). Conversely, correlates of men accurately suspecting their spouses of infidelity were: women being denied preferred sex position (OR, 0.50; 95\% CI: 0.26-0.98), couple being in polygamous marriage (OR, 2.51; 95\% CI: 1.01-6.23) and higher score on domestic violence - higher e scale (OR, 1.33; 95\% CI: 1.15-1.54).

Conclusion High rates of inaccurate infidelity suspicions in this community may be detrimental to marital relationships. Couples need to consider correlates that aid accurate suspicion before bring up the issue for discussion.

\section{P4.061 PHYSICAL ACTIVITY AND RISKY SEXUAL BEHAVIOUR IN MEN WHO HAVE SEX WITH MEN AND HETEROSEXUAL MALES IN TEL AVIV, ISRAEL}

doi:10.1136/sextrans-2013-051184.0959

'Z Mor, ${ }^{2} \mathrm{~K}$ Parfionov, ${ }^{2} \mathrm{~N}$ Davidovitch, ${ }^{3} \mathrm{G}$ Grotto. 'Ministry of Health, Ramla, Israel; ${ }^{2} \mathrm{Ben}$ Gurion University, Beer Sheva, Israel; ${ }^{3}$ Ministry of Health, Jerusalem, Israel

Background Men who have sex with men (MSM) who exercise intensive anaerobic training (IAT) become muscular and physicallyattractive, are increasing the number of potential sex-partners and their risk for STI/HIV. This study compared training practises between MSM and heterosexuals, and explored factors associating IAT with sexual-behaviour.

Methods This cross-sectional study included a convenient sample of men from 5 Tel-Aviv gyms, who completed anonymous questionnaires regarding their physical training, health and sexual behaviour. Trainees who exercised more than the median anaerobic traininghours were regarded as IAT, and those who performed $>$ one unprotected anal/vaginal-intercourse in the last six months were defined as sexual-risky.

Results In 2011, 184 (48\%) MSM and 197 (52\%) heterosexuals completed the questionnaire. MSM showed a stronger desire to become muscular than heterosexuals, reported more weekly hours of IAT (5.0 and 3.9, respectively, $p<0.001$ ) and used protein-powers or anabolic-steroids more commonly than heterosexuals. MSM reported that the main reasons for training were refining body-shape and improving self-confidence, while heterosexual indicated that weight-loss and health improvement were the main reasons for training. MSM were more sexual-risky than heterosexuals.

Of all sexual-risky, $61.9 \%(\mathrm{~N}=70)$ performed IAT, while $38.1 \%$ $(\mathrm{N}=43)$ perform moderate anaerobic training, $p<0.01$. This association was stronger among MSM than in heterosexuals $(p<0.01$ vs $p=0.05$, respectively). In multivariate analysis, MSM, younger age, dissatisfaction from body shape, strong desire to be more attractive, higher sexual obsession-score, and the use of protein supplement powders predicted an IAT The interaction between MSM and IAT among risky participants was multiplicative.

Discussion MSM practised more IAT than heterosexuals, and their interaction between IAT and sexual-risk was multifactorial. MSM community can benefit from holistic approach of sexualhealth, addressing body-image and IAT. The gym gay-culture demonstrates how internal dynamics and social norms are possible factors driving MSM to high-risk for HIV. 